\title{
Enhanced Learning with Abacus and its Analysis Using BCI Technology
}

\author{
Geeta $\mathbf{N}$ \\ Department of Computer Science \& Engg, KLE Dr. M.S.Sheshagiri CET, Udyambag, Belgaum-590008, India \\ geeta_malgi@yahoo.co.in \\ Rahul Dasharath Gavas \\ Department of Computer Science \& Engg, KLE Dr. M.S. Sheshagiri CET, Udyambag, Belgaum-590008, India \\ rahulgavas@gmail.com
}

\begin{abstract}
Although technology is successfully being used these days as a tool to improve education at all levels, its improper usage is curbing the imagination of the student community, leading to a diminution in their thinking capacity and ability to focus and concentrate. As attention is a vital cognitive feature of any learning process, students these days are not coping well with this process. This study attempts to analyse the focusing capacity of students from two different backgrounds; students who have undergone training in mental arithmetic and usage of the abacus and students without any formal mental arithmetic training. The analysis is done through a simple Electroencephalogram (EEG) based gaming software, which measures the time needed for the players to focus and reach a specific attention level. An EEG device measures brain invoked potentials. Due to the availability of low cost commercial grade EEG devices, usage of these devices today, is not confined only to research and clinical purposes, but is being used beyond these applications. This study is an attempt to apply Brain Computer Interface (BCI) Technology to assess cognition. The performance of the first category was found to be better than the second set of students.
\end{abstract}

Index terms-EEG, BCI, attention, abacus, cognition.

\section{INTRODUCTION}

Advancements in technology have affected our lives and made it very comfortable. Today, doing business, communicating with friends, gathering information and managing day to day jobs has been made very easy with just a single click. But the increased use of gadgets and web applications has reduced physical activity of its user in general and more specifically the student community. The overuse of the handheld gadgets and web applications is consuming all their valuable time and is even hampering their personal growth. Researchers have found out the following adversaries in today's children i) lack of patience ii) elevated exasperation iii) declining writing skills and iv) lack of physical interactivity.

The above observations are having a tremendous negative impact on the Learning Process in students as they are unable to focus their attention on a particular object for a longer time. Researchers are addressing various theoretical issues in cognitive psychology of learning and memory as these also have practical implications for education.

The main goal of learning is to increase intelligence, and a considerable amount of research has been done in this regard. Efforts to ascertain this using nutritious food [1] and education [2], styles of teaching in conjunction to learning styles [30] are remarkable. However improving intelligence by mere education is still controversial [3].

According to the scrutinized survey conducted in conjunction to explore the strategies involving cognitive aspects of enhancing the learning process [4], it can be categorized into three major paradigms: (1) Material distribution and practice, (2) Assessment of the extent of learning, (3) Interrogation by the students for themselves.

Numerous methodologies have been used to improvise learning. With the advent of computers, graphics have been used as a powerful tool in teaching [5]. As a traditional model, conversational way of learning, called the Pask's model is stressed in [6]. Many attempts have been made to access the relationship between teaching and learning effectiveness. One such method is by Student Evaluation of Teaching (SET) [7]. Collaboration is yet another powerful way of learning because it forms a social structure which culminates into interaction and exchange of views [8]. The principle of do-and-learn, also called learning through experience gives a clear understanding of the concepts which overtakes conventional method of teaching [9]. It is observed that the Virtual Classroom (VCR) is an effective means of education when compared to traditional classroom teaching [11]. In all these attempts made, the main underlying requirement is attention.

BCI based games have paved a new dimension in attention enhancement using neurofeedback where users control a specific game using controlled thought processes [10-12,14].

The present study compares the brain dynamics of the subjects involved in mental arithmetic practices using abacus with that of non-abacus practitioners. It uses Brain-Computer Interface technology $[15,16,29]$ to compare the attention levels of the two categories of users. It was found that abacus proficient subjects exhibit 
outstanding capability in mental calculation which may be the result of higher concentration levels. Fig.1 shows the schema of the implementation using Brain Computer Interface. The first phase deals with acquiring the brain waves using an EEG device. This is followed by a phase dealing with signal processing algorithms to extract data from the raw brain waves and then a phase dealing with the translation of the data into a machine understandable format. Finally this is used to drive computer applications.

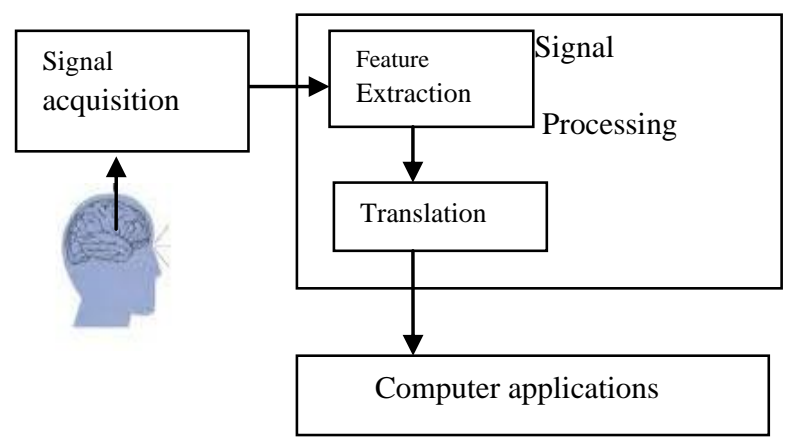

Fig.1. Block diagram of BCI.

\section{A. Attention}

According to the clinical model, attention implies focusing on a single facet while ignoring the rest. Attention has also been referred to as the allocation of processing resources [17]. It is deep rooted in psychology and neuroscience. With the advancements in technology, efforts have been made to develop relational schemas which govern their correlation with respect to electric impulses in the brain [18-21]. This aids in driving many real time applications. There are two aspects of the response of the human brain to the external environment [22] one is bottom-up processing or stimulus driven or exogenous attention. It could be a response to sudden noises. The other one is top-down processing or goal driven or endogenous or executive attention. It is under the control of the person who is attending it. The exogenous aspects of attention involve parietal and temporal cortices and brainstem and the endogenous aspects involve frontal cortex and basal ganglia [23-25].

Multimedia multitasking is another behavior observed in the younger generations today. This has resulted due to the rapid changes in mobile computing and Internet access environments [26]. Multitasking can be defined as the attempt to perform two or more tasks simultaneously. Research shows that when multitasking, people make more mistakes or perform their tasks slowly [27] as attention must be divided among all the components of the task to perform them. Research also shows that multitasking affects the way of human thinking, learning, retention, and other information processing acts [25].

\section{B. Abacus}

Though its uses have ceased considerably in this computer age, it has still been a popular tool which bestows the users, the power of both manipulating the tool in its physical as well as mental form. This can involve calculations which frequently take 10 digits with significant speeds and precision [12]. This is accomplished by the finger movements in the initial stages which are replaced by mental calculations in the later levels of training. This is followed by the amalgamation of several cognitive features like momentary storage of transitional data, number identification, and quick manipulation. It is found that abacus experts possess higher levels of attention which is vital to achieve anything.

The overall structure of the paper is organized as follows: The initial part of the current study includes a survey of challenges faced in modern educational systems and work done towards overcoming them. The need of cognition to improvise the learning process is identified and abacus as one of the tools to catalyze it is discussed. The implementation deals with monitoring the attention state of the abacus and non-abacus literates which is given in Section II. A comparison of the data sets of both classes of subjects is presented graphically in Section III. The conclusion and future scope of the study are given in Section IV and V respectively.

\section{IMPLEMENTATION}

The Human brain is composed of millions of neurons, which eject electrons at unparalleled paces. The implementation focuses on BCI technology, a new research area in the field of computer science. The technology revolves around the extraction of brain generated signals to operate computer applications

The algorithms used in the Electroencephalogram (EEG) device used to generate digital data corresponding to different brain states including attention and meditation. The waveforms depict various mental and physical processes underlying in humans. During signal processing, the unwanted parts of the waveforms called artifacts, which culminate due to accidental eye blinks, muscle movements, respiration, etc., are removed. The pure waveform obtained is used to study the various physiological processes and is used to drive an application or to detect mental disorders.

Based on frequency, the brain waves are categorized into lower and higher alpha, lower and higher beta, delta, theta, gamma, etc. Each waveform depicts a particular state of mind and body. Many animals, including humans produce gamma waves $(40-60 \mathrm{~Hz})$ when focusing attention on a particular object or activity [28].

In this study, the data corresponding to attention state waveforms were collected from people of different age groups in time domain with the aid of an EEG device from Neurosky.

Subjects within the age groups from 6 to 22 were chosen. All the subjects were healthy and the experiment was conducted in normal environmental conditions. Two types of subjects were enrolled into the study, one type comprised of abacus experts and others were not. The abacus literate subjects were asked to solve some arithmetic problems which were dictated orally until the BCI game ended. The non abacus practitioners were 
asked to play a simple BCI game developed using OpenGl (Open Graphics Library)-an Application Program Interface (API) for rendering 2D/3D graphics. Fig. 2 shows a snapshot of the BCI game developed for this purpose. The user had to move a sphere from source to destination and the movement was based upon the attention values. A few numbers of intervening random figures were used to distract the user and curb his attention and add to the difficulty level of the game. The lighting, shading and material properties were randomized to facilitate this. In both the cases, subject's brain data was captured using Neurosky Mindwave Mobile EEG [13].

\section{A. Hardware and software details}

The device used is a single channel acquisition, wireless EEG, with the sensor capturing the waveforms safely from the frontal cortex region. The device uses dry sensor technology. Software named ThinkGear Connector (TGC) is used to connect the EEG to the computer and the data transfer takes place through the Bluetooth device. TGC runs as a background process and directs the data from the headset to an open network socket from the serial port. For every second, the device computes and measures the attention eSense measure. Internally, it considers electric signals (Gamma waveforms) to accomplish this. The analog data is then translated into binary form. This can be retrieved using the TG_GetValue (connectionId, TG_DATA_ATTENTION) api. The first argument specifies the connection status of the EEG device and the second parameter is used to get the attention values in percentages.

The overall testing schema can be summarized with the aid of the following algorithm:

\section{B. Algorithm}

Input: Brain waves of subjects involved in mathematical computations.

Output: Percentage of attention retaining capacity above a certain threshold.

Procedure:

1. Connect the EEG to the system.

2. If successful connection,

\{

Read brain wave data

$\mathrm{n}=$ number of abacus knowing subjects;

$\mathrm{m}=$ non abacus subjects;

$\mathrm{t}=$ time taken to solve a problem;

counter $=0$;

$/ /$ structure for data storage

struct

\{

int attention[t];

float percentage;

\}abacus[n], non_abacus[m];

\}

//connection not successful

else go to step 1.
3. Calculate attention retaining capability of abacus subjects

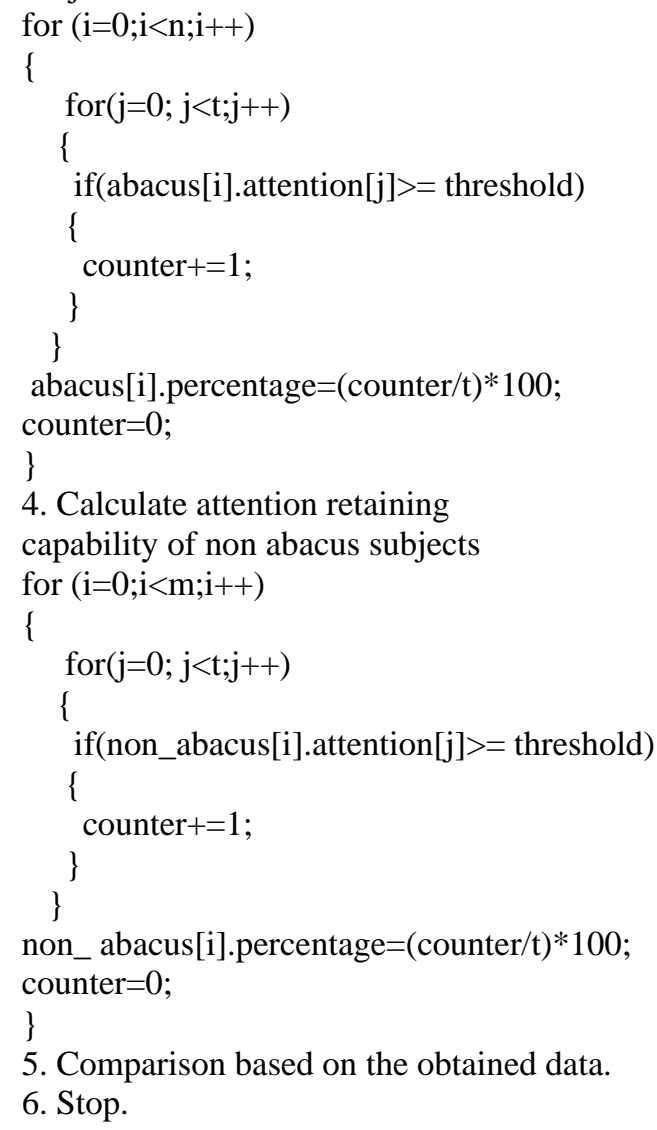

C. Programming modules used in the BCI game. main() \{

//Connect the EEG errorCode $=($ int $)$ (check connection $)$ if errorCode $<0$ return Connection failure $\mathrm{p} \leftarrow$ initial screen position Drawscene(); \} Reshape();

\section{Drawscene()}

\{

//Clear the colour and depth buffers and set the //model view matrix

$$
\begin{aligned}
& \text { glLoadIdentity(); } \\
& \text { R Random Number }
\end{aligned}
$$

//Set the material properties including ambience, //diffuse, specular and shininess //Set the light position and light diffuse API's //Randomize the above API's with R passed as //parameter MoveBall(); DrawCircle(); //Swap the buffers \} 


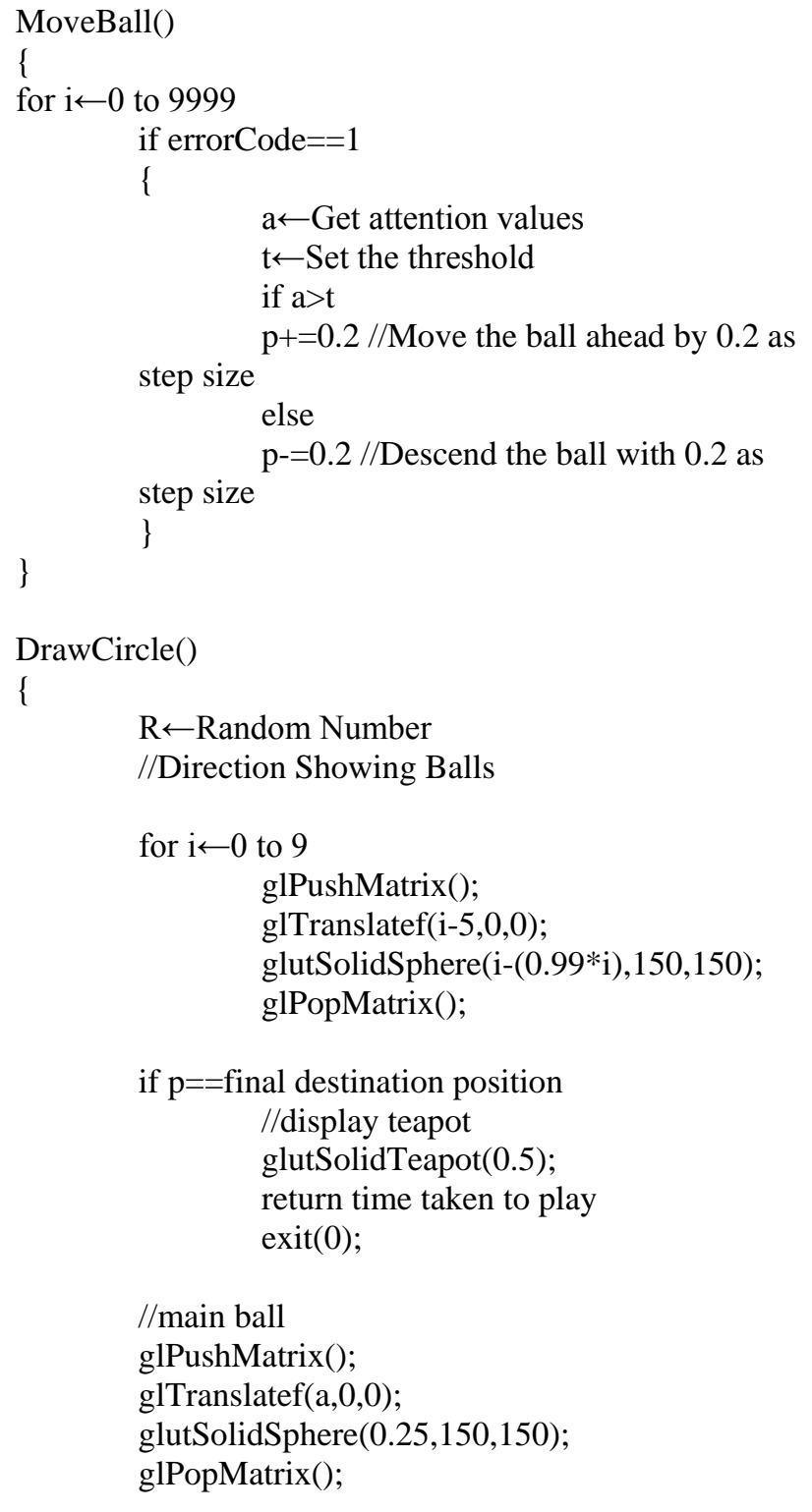

//similarly draw random balls with $\mathrm{R}$ passed as parameter in it

$/ / \mathrm{R}$ should be used in glTranslate(...) and glutSolidSphere (...) to randomize their movement and shape

\}

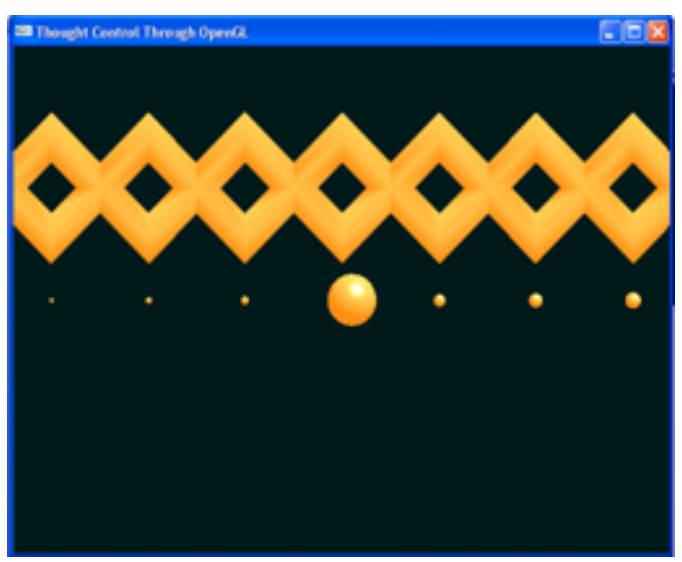

Fig. 2. Snapshot of BCI game developed using OpenGl.

\section{RESUlTS AND DisCUSSIONS}

The BCI game was developed for non -abacus practitioners using OpenGl graphics package. The nonabacus practitioners were made to play the game and simultaneously their eSense measures were recorded. In case of abacus literates, the students were asked to solve arithmetic problems mentally and their eSense measures were recorded through the game. The brain data was read from an assortment of subjects and the data sets were manipulated using the algorithm mentioned in section II.B and the results are as shown in Fig. 3 through 4, for abacus and non abacus students, respectively.

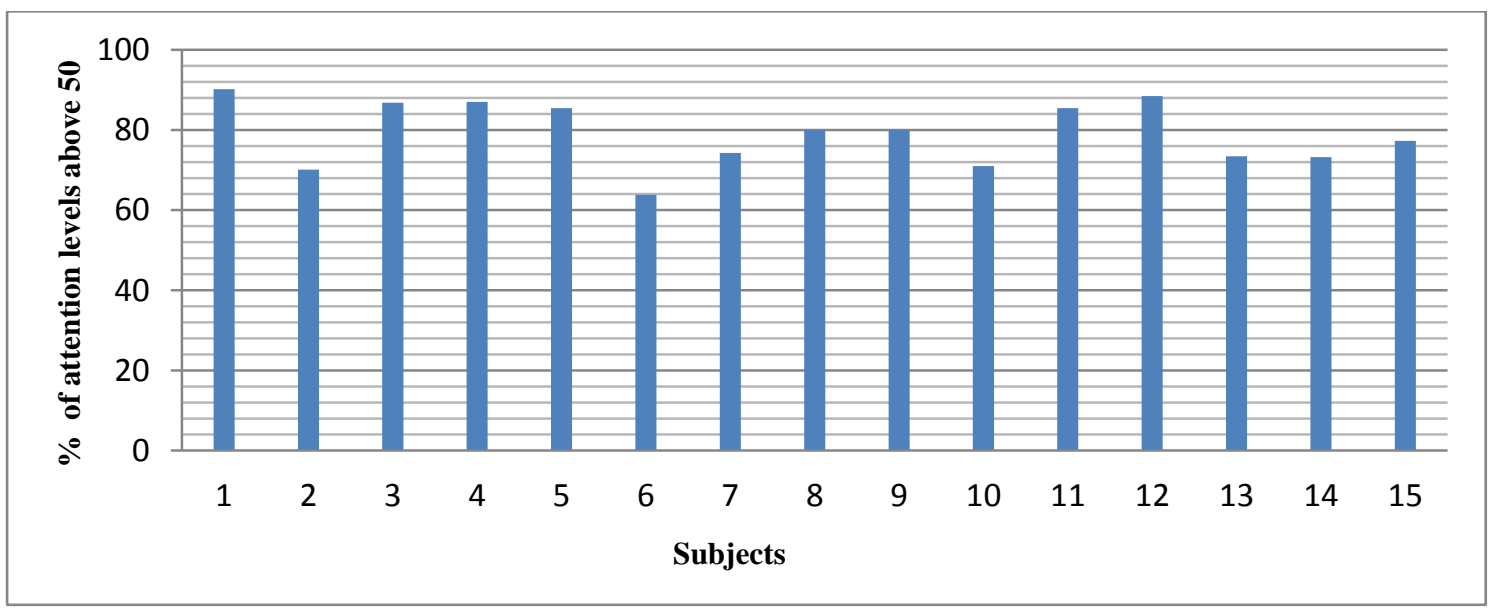

Fig. 3. Graph showing the percentage of attention levels above $50 \%$ in abacus literates 


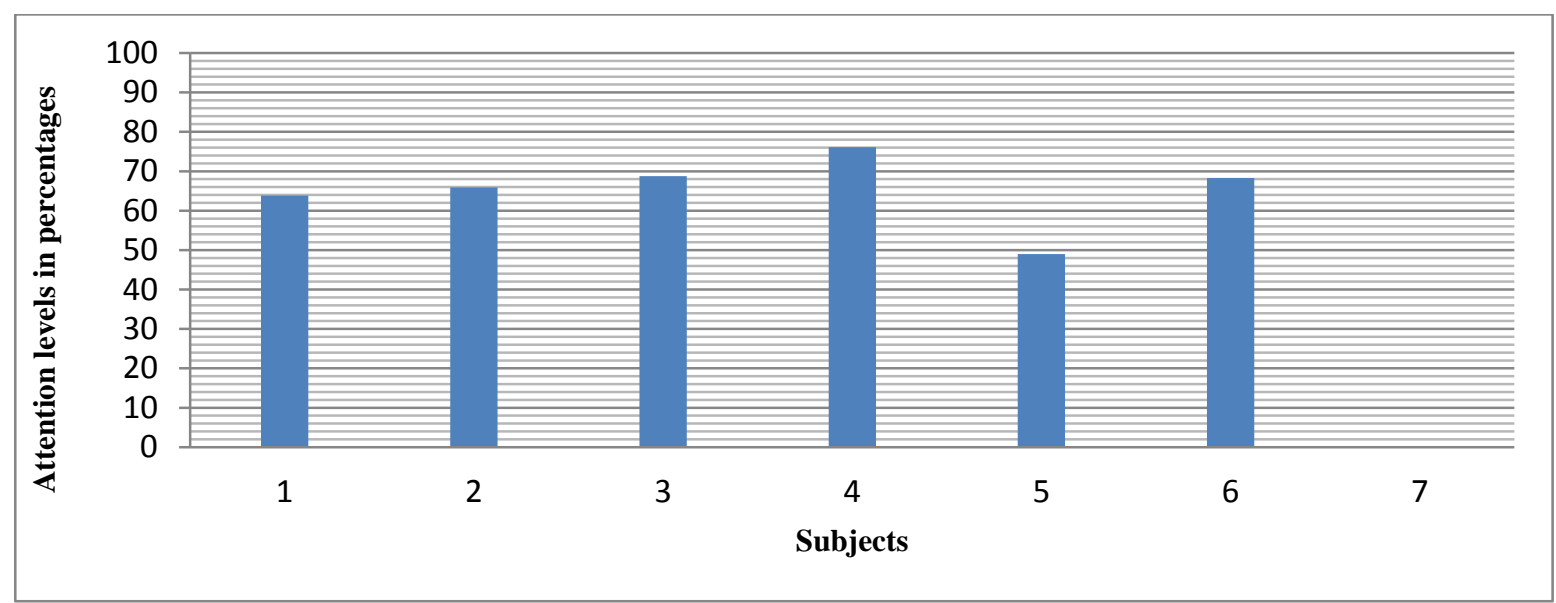

Fig. 4. Graph showing the percentage of attention levels above $50 \%$ in non - abacus practitioners.

The results obtained show how well the abacus students excelled the rest in consistently maintaining their attention value above a certain threshold, which was $50 \%$ in this case.

\section{CONCLUSION}

Abacus instills the capability of retaining optimum attention which is evident from the results obtained. Attention values show drastic rise and falls and is spare, in subjects who are not abacus literates. This shows how a simple yet powerful mental calculation technique, plays a major role in fostering the attention, which is crucial to pursue any education. Also, the study shows how an EEG device can effectively measure the attention levels in an individual and thus aid him/her to rectify his concentration skills.

\section{Future SCOPE}

The study urges the need to include abacus as a part of the curriculum in all aspects of learning. This highly enhances the outcome of education. Learning institutes can use EEGs to assess the improvements and attention levels in students. This paves a way towards effective learning.

\section{ACKNOWLEDGEMENTS}

The authors are immensely grateful for the valuable guidance provided by Dr. Nandini Sidnal, HOD, Department of Computer Science \& Engg, KLE Dr. M.S. Sheshagiri CET, Udyambag, Belgaum.

\section{REFERENCES}

[1] Benton,D.(2001),"Micro-nutrient supplementation and the intelligence of children", Volume 25, Issue 4, June 2001, Pages 297-309, Elsevier.

[2] Ceci, S. J. (1991), "How much does schooling influence general intelligence and its cognitive components?", A reassessment of the evidence. Developmental Psychology, 27, 703-722. doi: 10.1037/0012-1649.27.5.703
[3] Paul Irwing, Alya Hamza, Omar Khaleefa, Richard Lynn, "Effects of Abacus training on the intelligence of Sudanese children", Personality and Individual Differences 45(2008)694-696, Elsevier. doi:10.1016/j.paid.2008.06.011.

[4] Henry L. Roediger III, Mary A. Pyc, "Inexpensive techniques to improve education: Applying cognitive psychology to enhance educational practice", Journal of Applied Research in Memory and Cognition 1 (2012) 242 248, Elsevier. doi: 10.1016/j.jarmac.2012.09.002.

[5] Mary Hegarty, "Dynamic visualizations and learning: getting to the difficult questions", Learning and Instruction 14(2004)343-351, Elsevier. doi:10.1016/j.learninstruc.2004.06.007.

[6] Bernard Scott, "Gordon Pask's Conversation Theory: A Domain Independent Constructivist Model of Human Knowing”, Foundations of Science, special issue on "The Impact of Radical Constructivism on Science",edited by A. Riegler, 2001, vol. 6, no.4: 343-360.

[7] Mariyamni Awang, Balbir Singh, Iskandar Dzulkarnain, "An Analysis of the Relationship between Effective Teaching and Effective Learning at UTP", Procedia Social and Behavioral Sciences 56 (2012) 594 - 601, Elsevier. doi: 10.1016/j.sbspro.2012.09.693.

[8] DILlENBOURG, P., BAKER, M., BLAYE, A. \& O'MALLEY, C., "The evolution of research on collaborative learning”, In E. Spada \& P. Reiman (Eds) Learning in Humans and Machine: Towards an interdisciplinary learning science. (Pp. 189-211). Oxford: Elsevier.

[9] Orazio Miglino, Alberto Venditti, Alessandra Delli Veneri, Andrea Di Ferdinando, "Eutopia-Mt. teaching mediation skills using multiplayer on-line role playing games", Procedia Social and Behavioral Sciences 2 (2010) 2469-2472, Elsevier. doi:10.1016/j.sbspro.2010.03.355.

[10] Thomas, Kavitha P Vinod, A.P.; Guan, Cuntai, "Design of an online EEG based neurofeedback game for enhancing attention and memory", Engineering in Medicine and Biology Society (EMBC), 2013 35th Annual International Conference of the IEEE. doi: 10.1109/EMBC.2013.6609529.

[11] Das, S., Dhande, A.P. ; Bhoi, M., "Leveraging Virtual Classroom for effective learning", Technology for Education (T4E), 2010 International Conference, IEEE. doi: 10.1109/T4E.2010.5550051.

[12] Stigler, J.W., 1984. "Mental abacus:" the efects of abacus training on Chinese children's mental calculation", Cogn. 
Psychol. 16, 145-176. doi: 10.1016/0010-0285(84)900069

[13] Available at store.neurosky.com/products/mindwavemobile.

[14] Qiang Wang, Olga Sourina, and Minh Khoa Nguyen, "EEG-based "Serious" Games Design for Medical Applications",2010 International Conference on Cyberworlds, IEEE doi: 10.1109/CW.2010.56.

[15] Jonathan R. Wolpaw, et.al., "Brain-Computer Interface Technology: A Review of the First International Meeting", IEEE TRANSACTIONS ON REHABILITATION ENGINEERING, VOL. 8, NO. 2, JUNE 2000. doi=10.1.1.331.2636.

[16] Gerwin Schalk, et.al., "BCI2000: A General-Purpose Brain-Computer", IEEE TRANSACTIONS ON BIOMEDICAL ENGINEERING, VOL. 51, NO. 6, JUNE 2004.

[17] Anderson John R. (2004) Cognitive psychology and its implications $\left(6^{\text {th }}\right.$ ed) Worth Publishers. P.159 ISBN 9780-7-167-0110-1.

[18] Fred Travis, Jonathan Shear, "Focused attention, open monitoring and automatic self -transcending: Categories to organize meditations from Vedic, Buddhist and Chinese traditions", Consciousness and Cognition xxx (2010) xxx-xxx, Elsevier.

[19] Georgios Patsis, Hichem Sahli, Werner Verhelst, Olga De Troyer, "Evaluation of Attention Levels in A Tetris Game Using a Brain Computer Interface", User Modeling, Adaptation, and Personalization Lecture Notes in Computer Science Volume 7899, 2013, pp 127-138, Springer Link. doi: 10.1007/978-3-642-38844-6_11.

[20] Zhijiang Wan, Jian He, Alicia Voisine, "An Attention Level Monitoring and Alarming System for the Driver Fatigue in the Pervasive Environment", Brain and Health Informatics Lecture Notes in Computer Science Volume 8211, 2013, pp 287-296, Springer Link. doi: 10.1007/978-3-319-02753-1_29.

[21] Suzanne E. Hidi, "A reexamination of the role of attention in learning from text", Educational Psychology Review December 1995, Volume 7, Issue 4, pp 323-350, Springer Link. doi: 10.1007/BF02212306.

[22] Theeuwes J. (1991) Exogenous and Endogenous control of attention - the effect of visual onsets and offsets. Perception and Psychophysics 49(1): 83 - 90.

[23] Posner, M. I. \& Petersen, S. E. (1990) The attention system of the human brain. Annual Review of Neuroscience 13: 25-42. doi: 10.1146/annurev.ne.13.030190.000325.

[24] Posner, M.I. \& Rothbart, M.K. Attention, self-regulation and consciousness. Phil. Trans. R. Soc. Lond. B (1998) 353, 1915-1927.

[25] Posner, M. I. (1980) Orienting of attention. Quarterly Journal of Experimental Psychology 32(FEB): 3-25. doi: 10.1080/00335558008248231.

[26] "Analysis of Youngsters' Media Multitasking Behaviors and Effect on Learning", International Journal of Multimedia and Ubiquitous Engineering Vol. 8, No. 4, July, 2013.

[27] Margaret W. Matlin (2013). Cognition (Textbook) (8 ed.). Wiley. ISBN 978-1-118-14896-9.

[28] Kaiser J. Lutzenberger W (2003) "Induced gamma- band activity and human brain function' Neuroscientist 9 (6): 475-84. doi:10. 1177/1073858403259 137. PMID 14678580 .

[29] YI, Fang, Hao LI, and Xiaojie JIN. "Improved Classification Methods for Brain Computer Interface
System." March 2012 in MECS, doi: 10.5815/ijcnis.2012.02.03.

[30] Abbas Pourhosein Gilakjani, "A Match or Mismatch Between Learning Styles of the Learners and Teaching Styles of the Teachers", I.J.Modern Education and Computer Science, 2012, 11, 51-60. doi: 10.5815/ijmecs.2012.11.05.

\section{Authors' Profiles}

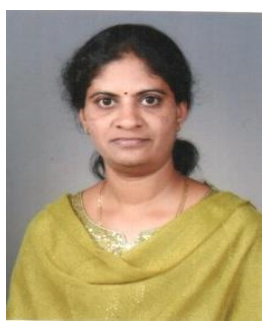

Prof Geeta N. has graduated in Electronics and Communication Engineering from GIT, Belgaum and completed Post-graduation in Computer Networking from VTU Belgaum. Currently she is working as Assoc. Prof in Computer Science and Engineering at KLE Dr. M.S. Sheshagiri College of Engineering and Technology, Belgaum, Karnataka. Her areas of interest include cognitive computing, brain-computer interface, computer networks and parallel processing.

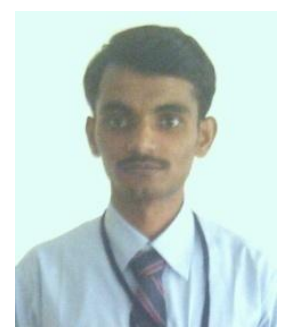

Mr. Rahul Dasharath Gavas is pursuing his Bachelor of Engineering in Computer Science and Engineering from KLE Dr. M.S.Sheshagiri College of Engineering and Technology. His areas of interest include cognitive computing, brain-computer interface and unpredictability theory. 